\title{
Effects of the brown rice diet on visceral obesity and endothelial function: the BRAVO study
}

\author{
Michio Shimabukuro ${ }^{1,2,3,4 *}$, Moritake Higa ${ }^{3,4}$, Rie Kinjo5 ${ }^{5}$, Ken Yamakawa ${ }^{3,4}$, Hideaki Tanaka ${ }^{4}$, \\ Chisayo Kozuka ${ }^{3}$, Kouichi Yabiku ${ }^{3}$, Shin-Ichiro Taira ${ }^{3}$, Masataka Sata ${ }^{2}$ and Hiroaki Masuzaki ${ }^{3}$ \\ ${ }^{1}$ Department of Cardio-Diabetes Medicine, The University of Tokushima Graduate School of Health Biosciences, \\ 3-18-15 Kuramoto, Tokushima 770-8503, Japan \\ ${ }^{2}$ Department of Cardiovascular Medicine, The University of Tokushima Graduate School of Health Biosciences, \\ 3-18-15 Kuramoto, Tokushima 770-8503, Japan \\ ${ }^{3}$ Division of Endocrinology, Diabetes and Metabolism, Haematology, Rheumatology, Second Department of Internal \\ Medicine, Graduate School of Medicine, University of the Ryukyus, Okinawa, Japan \\ ${ }^{4}$ Diabetes and Lifestyle-Related Disease Center, Tomishiro Central Hospital, Okinawa, Japan \\ ${ }^{5}$ Division of Clinical Laboratory, Tomishiro Central Hospital, Okinawa, Japan
}

(Submitted 28 January 2013 - Final revision received 27 June 2013 - Accepted 27 June 2013 - First published online 12 August 2013 )

\begin{abstract}
Brown rice (BR) and white rice (WR) produce different glycaemic responses and their consumption may affect the dietary management of obesity. In the present study, the effects of BR and WR on abdominal fat distribution, metabolic parameters and endothelial function were evaluated in subjects with the metabolic syndrome in a randomised cross-over fashion. In study 1, acute postprandial metabolic parameters and flow- and nitroglycerine-mediated dilation (FMD and NMD) of the brachial artery were determined in male volunteers with or without the metabolic syndrome after ingestion of either BR or WR. The increases in glucose and insulin AUC were lower after ingestion of BR than after ingestion of WR $(P=0.041$ and $P=0.045$, respectively). FMD values were decreased 60 min after ingestion of WR $(P=0.037 v$. baseline), but the decrease was protected after ingestion of BR. In study 2 , a separate cohort of male volunteers $(n$ 27) with the metabolic syndrome was randomised into two groups with different BR and WR consumption patterns. The values of weight-based parameters were decreased after consumption of BR for 8 weeks, but returned to baseline values after a WR consumption period. Insulin resistance and total cholesterol and LDL-cholesterol levels were reduced after consumption of BR. In conclusion, consumption of BR may be beneficial, partly owing to the lowering of glycaemic response, and may protect postprandial endothelial function in subjects with the metabolic syndrome. Long-term beneficial effects of BR on metabolic parameters and endothelial function were also observed.
\end{abstract}

Key words: Glycaemic indices: Endothelial function: Obesity

Rice is generally consumed after it is refined to remove the outer bran and germ portions of the intact grains (i.e. brown rice, BR) and produce white rice (WR), consisting primarily of the starchy endosperm. The postprandial blood glucose responses elicited on consumption of WR or BR are substantially different ${ }^{(1-3)}$. Whole grains have been shown to be associated with a low fasting insulin level and a low glycaemic response after meal ingestion $^{(4-6)}$. The intake of whole grains, but not of refined grains, has also been reported to be associated with low body weight and adiposity ${ }^{(5,6)}$. Thus, the intake of whole-grain BR might be beneficial for controlling weight and obesity. Recently, we have reported that $\mathrm{BR}$ and $\gamma$-oryzanol, one of its major components, improve high-fat diet-induced metabolic derangement and attenuate the preference for dietary fat, by decreasing hypothalamic endoplasmic reticulum stress ${ }^{(7)}$. Therefore, we hypothesised that BR may be useful for ameliorating obesity and the metabolic syndrome, a constellation of obesity-based metabolic abnormalities (glucose intolerance, insulin resistance, dyslipidaemia and hypertension, all well-documented risk factors of CVD), by regulating eating behaviour. Until now, clinical trials have not been conducted to provide evidence of a direct effect of BR on body weight and eating behaviour.

\footnotetext{
Abbreviations: BR, brown rice; FMD, flow-mediated dilation; GI, glycaemic index; GLP, glucagon-like peptide; HOMA-IR, homeostasis model assessment of insulin resistance; hs-CRP, high-sensitivity C-reactive protein; NMD, nitroglycerine-mediated dilation; SFA, subcutaneous fat area; VFA, visceral fat area; WR, white rice.
} 
Furthermore, an inverse relationship between the intake of whole grains and the risk of IHD has been reported by two cohort studies $^{(8,9)}$. Although such protective effects are usually explained by the presence of various constituents, such as dietary fibre, phytic acid and vitamins, there is a lack of concrete evidence supporting these assumptions ${ }^{(10)}$. A clinical metaanalysis has shown an association between postprandial glucose levels and macrovascular complications in non-diabetic and diabetic individuals ${ }^{(11)}$. Our group ${ }^{(12)}$ and others ${ }^{(13)}$ have reported that postprandial glucose elevation causes endothelial dysfunction, an early marker of atherosclerotic changes and a surrogate marker of future cardiovascular events. Such postprandial endothelial dysfunction has been suggested to be involved in vascular complications in diabetic ${ }^{(14)}$ and obese patients ${ }^{(15)}$. The inhibition of postprandial glucose elevation by whole grains might be beneficial for postprandial endothelial function, but a study examining such a relationship has not been reported.

The present investigative study was conducted to determine the acute effects of BR, when compared with those of WR, on postprandial metabolic parameters and postprandial endothelial function as well as the chronic effects of BR and WR consumption on abdominal fat distribution, metabolic parameters and endothelial functions in subjects with the metabolic syndrome.

\section{Study design and methods}

\section{Study design}

Healthy male volunteers were recruited by public advertisement from 1 December 2008 to 31 January 2009, and they underwent a $75 \mathrm{~g}$ oral glucose tolerance test ${ }^{(16)}$. In study 1 , eleven subjects participated, and in study 2 , twenty-seven subjects participated, as described below. A subject was defined as having the metabolic syndrome $e^{(17)}$ if he was obese (according to the modified Japanese criteria, having a waist circumference $\geq 85 \mathrm{~cm}$ ) and any two of the following four factors: (1) hypertriacylglycerolaemia (serum TAG concentration $\geq 1500 \mathrm{mg} / \mathrm{l}$ ( $1.69 \mathrm{mmol} / \mathrm{l})$ ); (2) low level of HDL-cholesterol (serum HDL-cholesterol concentration $<400 \mathrm{mg} / 1(1.04 \mathrm{mmol} / \mathrm{l})$ ); (3) elevated blood pressure (systolic blood pressure $\geq 130 \mathrm{mmHg}$ and/or diastolic blood pressure $\geq 85 \mathrm{mmHg}$ ); (4) high level of fasting glucose (serum glucose concentration $\geq 1000 \mathrm{mg} / \mathrm{l}(5.6 \mathrm{mmol} / \mathrm{l})$ ). Anthropometric measurements were taken while the subject was standing erect, including those of subcutaneous fat area (SFA) and intraabdominal visceral fat area (VFA). These measurements were taken at the level of the umbilicus using a standardised method involving computed tomography, as described previously ${ }^{(18)}$. The study protocol was approved by the Ethical Committee of Tomishiro Central Hospital, and the study was carried out in accordance with the principles of the Declaration of Helsinki as revised in 2000. The subjects gave written informed consent before the start of the study. The subjects were obligated to report any serious or unexpected adverse events, whether or not they appeared related to the intervention, immediately to the principal investigator and/or the research ethics committee in order to ensure appropriate management. For study 2, in a priori analysis made by a power analysis application ( $G^{*}$ Power version 3.1.7 ${ }^{(19)}$ ), the required sample sizes to detect differences in body weight per kg were as follows: fourteen in each group at $0 \cdot 8$ of the power $(1-\beta$ error probability) and the $5 \%$ level of significance ( $\alpha$ error probability) by the WilcoxonMann-Whitney test and twenty-eight matched pairs at 0.8 of the power ( $1-\beta$ error probability) and the $5 \%$ level of significance ( $\alpha$ error probability). Thus, we employed fourteen samples in each group, a total of twenty-eight samples, in the present study. The Consolidated Standards of Reporting Trials Statement 2010 checklist and a flow diagram of the progress through the phases of a parallel randomised trial of two groups are provided in Supplementary materials 1 and 2 (available online). The study protocol was registered at the University Hospital Medical Information Network Clinical Trials Registry (UMIN-CTR registration no. UMIN000009989).

\section{Study 1: acute effects}

On two mornings, at least $14 \mathrm{~d}$ apart, five participants without and six with the metabolic syndrome ingested a $1883 \mathrm{~kJ}$ ( $450 \mathrm{kcal})$ meal $^{(13)}$, including an $837 \mathrm{~kJ}$ (200 kcal) meal of either BR or WR of Japonica variety. Blood samples were collected before and 1, 2 and $4 \mathrm{~h}$ after meal ingestion. At the same time points, flowmediated dilation (FMD) and nitroglycerine-mediated dilation (NMD) were determined using automated measurements of the brachial arterial lumen, as described below ${ }^{(20,21)}$.

\section{Study 2: chronic effects}

Participants with the metabolic syndrome ( $n$ 27) were instructed to ingest BR or WR of Japonica variety for 8 weeks as described below. The participants were randomised by a computer-generated random number table into either the BR group followed by the WR group (BR-WR, $n$ 14) or the WR group followed by the BR group (WR-BR, $n$ 13). The BR-WR group consumed BR for the first 8 weeks and WR for the next 8 weeks. The WR-BR group followed the reverse consumption protocol. We instructed the participants to follow regular dietary intake/exercise habits during the intervention period, except for consumption of delivered rice. On each day, after ingestion of BR or WR, the participants recorded their compliance to the study requirement and their level of satiety (scale 1-10: not satisfied to satisfied). Before and after completion of both 8-week terms, blood and urine samples were collected, and FMD and NMD were determined; oral glucose tolerance test and abdominal computed tomography were also performed. Because primary outcomes were the effects of BR/WR on metabolic parameters and endothelial function and the carry-over effect between BR-WR and WR-BR groups could be minimised to these parameters after 2 months of treatment, we had not included a washout period.

\section{Assessment of vascular function}

FMD and NMD were measured using a vascular ultrasound system equipped with an automatic edge-tracking system for two-dimensional lumen imaging (UNEXEF; UNEX), according to the established guidelines ${ }^{(20,21)}$. The measurements of FMD 
and NMD were taken by a single laboratory technician using a skilful and stable technique to avoid inter-technician variation. The correlation coefficient between two FMD measurements was $0 \cdot 86$, with a CV of $11 \cdot 2 \%^{(21)}$. The diameter of the brachial artery was measured, at rest, in the cubital region. Subsequently, the cuff was inflated to $50 \mathrm{mmHg}$ above the systolic blood pressure, held there for $5 \mathrm{~min}$ and deflated. The diameter at the same point of the artery was monitored continuously, and the maximum dilation, occurring 45-60s after deflation, was recorded. The measurement of NMD was taken after a $15 \mathrm{~min}$ interval to allow for vessel recovery. Sublingual glyceryl trinitrate $(75 \mu \mathrm{g})$ was administered, and the maximum dilation of the brachial artery, at the same point as for the measurement of FMD, was confirmed and measured by a plateau in the diameter of the artery, using real-time monitoring of the diameter of the artery, over a period of at least $1 \mathrm{~min}$ after the point of maximum dilation. FMD and NMD values were calculated as follows:

$$
\begin{aligned}
\text { FMD or NMD value }(\%)= & (\text { maximum diameter } \\
& - \text { diameter at rest }) \\
& \times 100 / \text { diameter at rest. }
\end{aligned}
$$

\section{Biochemical measurements}

Venous blood samples were collected in tubes without an anticoagulant or in tubes with EDTA sodium $(1 \mathrm{mg} / \mathrm{ml})$ or spray-dried $\mathrm{K}_{2}$ EDTA and DPP-4 protease inhibitors (Becton Dickinson) for the determination of the concentrations of active glucagon-like peptide (GLP)-1 as well as plasma glucose and serum total cholesterol, HDL-cholesterol, TAG, creatinine and glucose. The concentration of LDL-cholesterol was estimated using Friedewald's method ${ }^{(22)}$. The concentration of glycosylated $\mathrm{Hb}$ was measured using HPLC and that of insulin using chemiluminescent enzyme immunoassay. The value for the concentration of glycosylated $\mathrm{Hb}(\%)$ was converted to the National Glycohemoglobin Standardization Program levels ${ }^{(23)}$. The concentration of high-sensitivity C-reactive protein (hs-CRP) (N Latex CRP II) was determined using immunonephelometric methods. The concentration of GLP-1 was measured using an ELISA kit (Millipore) ${ }^{(24)}$. The plasma concentrations of amidated GLP-1(7-36) and GLP-1 (7-37) were measured using an antibody that was highly specific for the N-terminus of GLP-1 and did not react with GLP-1(9-36), GLP-2 or glucagon. Other blood component assays were conducted using standard methods. Plasma was immediately separated by centrifugation at $3000 \mathrm{rpm}$ at $4^{\circ} \mathrm{C}$ for $10 \mathrm{~min}$ and serum by centrifugation at $1000 \mathrm{rpm}$ at room temperature for $10 \mathrm{~min}$. Urine samples were collected in light-resistant tubes and used to measure the concentration of urinary 8-isoprostane, using an enzyme immunoassay kit (Assay Designs).

\section{Statistical analysis}

Values are expressed as means and standard deviations, unless otherwise indicated. Two-tailed paired/unpaired Student's $t$ test or one-way factorial ANOVA, followed by Bonferroni's post hoc comparisons, was used to compare inter-group or intra-group means for parametric data. For small-group comparison, a non-parametric Wilcoxon-Mann-Whitney test was employed. All analyses were performed using Jump version 10.0.1.1 software (SAS Institute). Differences were considered to be significant if the $P$ value was $<0 \cdot 05$.

\section{Results}

\section{Study 1: acute effects}

The ages of the subjects with or without the metabolic syndrome were similar. Body weight, BMI, waist circumference and blood pressure were higher in subjects with the metabolic syndrome (Table 1). In subjects with the metabolic syndrome, the concentrations of glucose and insulin at $120 \mathrm{~min}$ after ingestion of the $1883 \mathrm{~kJ}$ ( $450 \mathrm{kcal}$ ) meal were lower when the meal consisted of BR than when it consisted of WR (Fig. 1). The increase in glucose AUC ( $\left.\Delta \mathrm{AUC}_{\text {glucose }}\right)$ after meal ingestion was lower when $\mathrm{BR}$ was consumed than when WR was consumed (253 (SD 140) v. 346 (SD 116) $\min \times$ $\mathrm{mmol} / \mathrm{l}, \quad P=0.006)$, and the increase in insulin AUC $\left(\Delta \mathrm{AUC}_{\text {insulin }}\right)$ was also lower in participants consuming $\mathrm{BR}$ than in those consuming WR (62886 (SD 32251) v. 95580 (SD 55310) $\mathrm{min} \times \mathrm{pmol} / \mathrm{l}, \quad P=0 \cdot 044$ ). The concentrations of LDL, HDL and TAG were not different between the subjects consuming the different types of rice (Supplementary material 3, available online), but that of NEFA, 240 min after meal ingestion, was significantly lower in subjects ingesting BR. In subjects without the metabolic syndrome, the concentrations of glucose, insulin, NEFA, LDL-cholesterol or HDL-cholesterol and TAG, $\Delta \mathrm{AUC}_{\text {glucose }}$ and $\Delta \mathrm{AUC}_{\text {insulin }}$ were all comparable between those consuming BR and those consuming WR.

Among subjects without the metabolic syndrome, the degree of FMD of the brachial arterial diameter during reactive hyperaemia was not different at 60, 120 and $240 \mathrm{~min}$ after ingestion of $\mathrm{BR}$ or WR, when compared with baseline values (Fig. 2). However, in subjects with the metabolic syndrome, FMD values were decreased at $60 \mathrm{~min}$ after consuming WR ( $P=0.037 v$. baseline) and returned to baseline values by $120 \mathrm{~min}$. FMD values were not decreased after ingestion of BR. In both the groups, NMD values did not change after ingestion of either BR or WR.

\section{Study 2: chronic effects}

There were no statistical differences in the baseline characteristics between the subjects in the two groups (Table 2). Subject adherence to the diets and post-meal satiety scores were comparable between the different diets (Supplementary material 4, available online). In the BR-WR group, body weight, BMI and waist circumference were decreased by the end of the 8-week BR diet period and returned to baseline values by the end of the WR diet period. In the WR-BR group, body weight, BMI, waist circumference and systolic blood pressure were comparable with the baseline values by the end of the 8-week WR diet period, but waist circumference and systolic blood pressure were lower at the end of the 8-week BR diet period. As shown in Table 3, the homeostasis model assessment 
Table 1. General characteristics of study 1 subjects

(Mean values and standard deviations)

\begin{tabular}{|c|c|c|c|c|}
\hline \multirow[b]{2}{*}{ Parameters } & \multicolumn{2}{|c|}{ Metabolic syndrome- } & \multicolumn{2}{|c|}{ Metabolic syndrome + } \\
\hline & Mean & SD & Mean & SD \\
\hline$n$ & \multicolumn{2}{|c|}{5} & \multicolumn{2}{|c|}{6} \\
\hline Age (years) & 45 & 4 & 41 & 5 \\
\hline Body weight (kg) & $67 \cdot 4$ & $6 \cdot 1$ & $79 \cdot 2^{\star}$ & $17 \cdot 6$ \\
\hline BMI $\left(\mathrm{kg} / \mathrm{m}^{2}\right)$ & $23 \cdot 1$ & $1 \cdot 7$ & $28 \cdot 1^{*}$ & 4.3 \\
\hline Waist circumference (cm) & $81 \cdot 3$ & 7.5 & $93 \cdot 8^{*}$ & $9 \cdot 6$ \\
\hline Systolic blood pressure (mmHg) & 124 & 14 & $141^{*}$ & 10 \\
\hline Diastolic blood pressure $(\mathrm{mmHg})$ & 82 & 6 & $89^{\star}$ & 6 \\
\hline Pulse (beats/min) & 60 & 4 & 75 & 14 \\
\hline Glucose (mmol/l) & $5 \cdot 9$ & 1.4 & $6 \cdot 1$ & 1.4 \\
\hline Insulin (pmol/l) & 39 & 14 & $87^{*}$ & 37 \\
\hline HOMA-IR & 1.43 & 0.51 & $3.48^{*}$ & 1.29 \\
\hline $\mathrm{HbA}_{1 \mathrm{c}}(\mathrm{NGSP} \%)$ & 5.58 & 0.68 & $6 \cdot 15$ & 1.76 \\
\hline Total cholesterol (mmol/l) & $5 \cdot 34$ & 0.64 & 5.42 & 0.86 \\
\hline LDL-cholesterol ( $(\mathrm{mmol} / \mathrm{l})$ & 3.55 & 0.24 & 3.27 & 0.62 \\
\hline HDL-cholesterol (mmol/l) & 1.37 & 0.13 & 1.26 & 0.27 \\
\hline TAG (mmol/l) & 1.41 & 0.62 & $2 \cdot 48^{*}$ & 0.70 \\
\hline $\mathrm{NEFA}(\mathrm{mmol} / \mathrm{l})$ & 0.196 & 0.136 & 0.248 & 0.133 \\
\hline AST (IU/I) & 23 & 6 & 31 & 12 \\
\hline ALT (IU/I) & 30 & 12 & 49 & 30 \\
\hline$\gamma$-GTP (IU/I) & 34 & 25 & $80^{*}$ & 46 \\
\hline
\end{tabular}

of insulin resistance (HOMA-IR) and total cholesterol and LDL-cholesterol concentrations of the BR-WR group were decreased at the end of the 8-week BR diet period and returned to baseline values over the course of the WR diet period. In the WR-BR group, values of all the variables were comparable with those at baseline at the end of the WR diet period, but the HOMA-IR and LDL-cholesterol levels decreased over the course of the BR diet period. The concentrations of markers of radical oxygen species (isoprostane), inflammation (hs-CRP) and incretin (active GLP-1) were all comparable between the periods of $\mathrm{BR}$ or WR consumption (Table 2). As shown in Fig. 3(a) and (b), body weight and waist circumference were significantly lower than those at baseline, after consumption of the BR diet in both the groups. VFA was also significantly smaller following the BR diet phase of the study than following the WR phase of the study in both the groups; SFA remained comparable between the phases of the study. We did not observe significant differences in FMD values in the BR-WR and WR-BR groups, but could find a difference in the combined group, indicating a sample size effect. As shown in Fig. 4, FMD values increased significantly from baseline by the end of the BR diet phase of the study, but returned to baseline values during the WR diet phase. We determined Pearson's correlation coefficient, $r$, a measure of the strength and direction of the linear relationship between metabolic variables and FMD at baseline, after the 8-week BR diet period and after the 8-week WR diet period. After the 8 -week BR diet period, there was a significant negative correlation with HOMA-IR $(r 0.652, P=0.016)$ and a borderline correlation with VFA $(r$ 0.387, $P=0.062)$, but not with other metabolic parameters including waist circumference, BMI, insulin levels at 0-120 min, glucose levels at $0-120 \mathrm{~min}$ and lipid parameters. NMD values remained comparable with the baseline values throughout the study.

\section{Discussion}

The present study revealed that a single daily meal that included BR, when compared with that consisting of WR, resulted in decreased postprandial concentrations of insulin and glucose and prevented postprandial endothelial dysfunction in subjects with the metabolic syndrome. It also revealed that switching the staple food of subjects with the metabolic syndrome from WR to BR led to a decrease in body weight, systolic blood pressure, HOMA-IR, and total cholesterol and LDL-cholesterol levels and improved the endothelial function.

\section{Acute effects}

In the present study, the $\Delta \mathrm{AUC}_{\text {glucose }}$ and $\Delta \mathrm{AUC}_{\text {insulin }}$ after ingestion of the $1883 \mathrm{~kJ}$ ( $450 \mathrm{kcal}$ ) meal were lower in subjects with the metabolic syndrome who consumed BR than in those who consumed WR (Fig. 1). Glycaemic index (GI) is defined as the response to $50 \mathrm{~g}$ available carbohydrates from a food in relation to $50 \mathrm{~g}$ available carbohydrates from a control food, i.e. it is a relative measure of the glycaemic response to available carbohydrates in a food ${ }^{(2)}$. The high content of viscous fibre and various enzymatic inhibitors are believed to slow the digestion and absorption of whole grains such as BR, compared with refined grains such as WR, and, therefore, elicit smaller postprandial glucose responses and a reduced insulin demand ${ }^{(25)}$. Although GI vary in types of rice $^{(3)}$, there is a difference between BR and WR belonging 

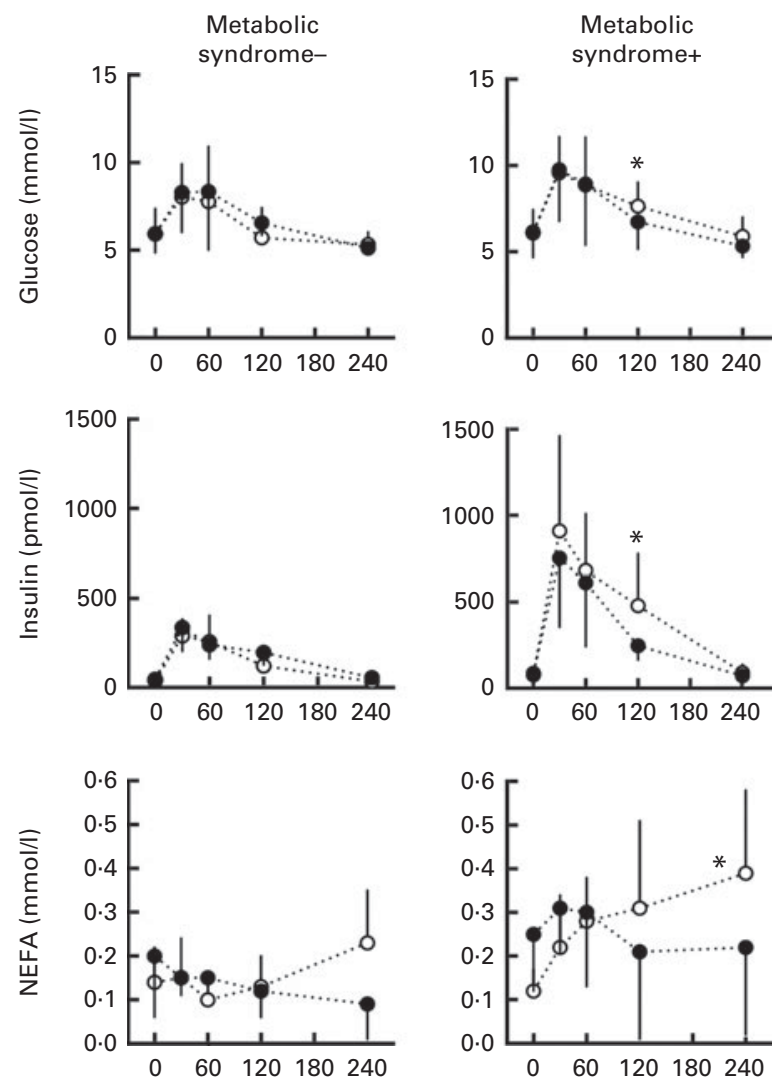

Time (min)

Fig. 1. Changes in biochemical parameters before and after ingestion of a meal with either brown rice $(B R, 0)$ or white rice (WR, $\bullet)$. On two mornings, participants with (BMI $\geq 25 \mathrm{~kg} / \mathrm{m}^{2}$ ) or without obesity (BMI $<25 \mathrm{~kg} / \mathrm{m}^{2}$ ) ingested either a $1883 \mathrm{~kJ}(450 \mathrm{kcal})$ meal, including an $837 \mathrm{~kJ}(200 \mathrm{kcal})$ meal of either BR or WR. Before and 60, 120 and 240 min after ingestion, blood samples were collected. The concentrations of glucose, insulin and NEFA are shown. For the concentrations of LDL-cholesterol, HDL-cholesterol and TAG, see Supplementary material 3 (available online). Values are means, with standard deviations represented by vertical bars. ${ }^{*}$ Mean value was significantly different from that at baseline $(P<0.05)$.

to the same type of rice. It has been reported that in the Japonica rice variety that we used, GI was significantly lower in BR than in WR (61.5 (SD 4.7) $v$. 75.9 (SD 6.6), $P<0.05$ ) when compared with a control $25 \%$ weight/volume glucose solution ${ }^{(26)}$. Notably, the effects of BR on postprandial glucose and insulin levels were observed only in subjects with the metabolic syndrome. A cross-over study involving overweight subjects has reported that their insulin sensitivity improved after being on a whole-grain diet for 6 weeks, as opposed to a refined-grain diet, independent of body weight ${ }^{(27)}$. Although BR has not been conclusively demonstrated to improve postprandial insulin action after consumption of a single meal, the observed decrease in postprandial NEFA levels in subjects with the metabolic syndrome may support this notion.

FMD, as assessed in the present study, mimics the NOmediated vasodilation produced by increased blood flow after a period of ischaemia. Non-endothelium-dependent dilation is useful in the measurement of the arterial changes induced by the administration of a sublingual dose of nitroglycerine, which predominantly reflects the smooth muscle response ${ }^{(20)}$. In subjects with the metabolic syndrome, FMD values of the brachial arterial diameter were decreased at 60 min after consumption of WR $(P=0.037 v$. baseline). Since NMD values did not change after WR ingestion, this change is largely due to postprandial endothelial dysfunction and not due to smooth muscle cell dysfunction. $\mathrm{We}^{(13)}$ and others ${ }^{(12)}$ have reported that patients with type 2 diabetes mellitus or impaired glucose tolerance and endothelial function, but not with impaired smooth muscle cell function, have worse endothelial function after meal consumption. The endothelial function in subjects with the metabolic syndrome may be impaired, to some degree, by hyperglycaemia and a consequent increase in reactive oxygen species levels ${ }^{(14)}$. We had previously reported that a reduction in postprandial blood glucose levels by $\alpha$-glycosidase inhibitor, which delays glucose release from complex carbohydrates, improves postprandial endothelial function in type 2 diabetes mellitus patients ${ }^{(28)}$. Although the exact mechanisms of the beneficial effects of BR on postprandial endothelial function are unknown, part of the BR protective function may result from the inhibition of postprandial glucose increase after consumption of a single meal.
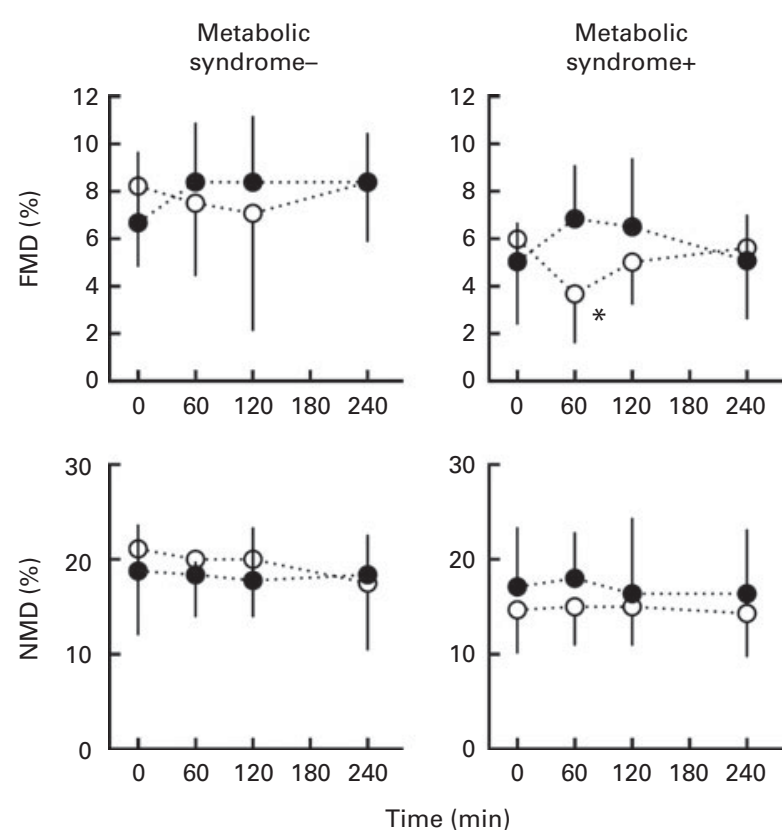

Fig. 2. Changes in forearm flow-mediated dilation (FMD) and nitroglycerinemediated dilation (NMD) values before and after ingestion of a meal with either brown rice $(\mathrm{BR}, \mathrm{O})$ or white rice $(\mathrm{WR}, \bullet)$. On two separate mornings, participants with $\left(\mathrm{BMl} \geq 25 \mathrm{~kg} / \mathrm{m}^{2}\right.$ ) or without obesity $\left(\mathrm{BMI}<25 \mathrm{~kg} / \mathrm{m}^{2}\right.$ ) ingested a $1882.8 \mathrm{~kJ}(450 \mathrm{kcal})$ meal, including an $836.8 \mathrm{~kJ}(200 \mathrm{kcal})$ meal of either BR or WR. Before and 60, 120 and $240 \mathrm{~min}$ after ingestion, FMD and NMD were measured using a novel vascular ultrasound system equipped with an edge-tracking system for two-dimensional imaging and automatic measurement. FMD and NMD values were calculated as follows: FMD or NMD value $(\%)=$ (maximum diameter - diameter at rest $) \times 100 /$ diameter at rest. Values are means, with standard deviations represented by vertical bars. * Mean value was significantly different from that at baseline $(P<0.05)$. 
Table 2. General characteristics of study 2 subjects

(Mean values and standard deviations)

\begin{tabular}{|c|c|c|c|c|c|c|c|c|c|c|c|c|}
\hline \multirow[b]{3}{*}{ Parameters } & \multicolumn{6}{|c|}{ BR-WR group ( $n$ 14) } & \multicolumn{6}{|c|}{ WR-BR group ( $n$ 13) } \\
\hline & \multicolumn{2}{|c|}{ Baseline } & \multicolumn{2}{|c|}{$\begin{array}{l}\text { 8-week BR } \\
\text { phase }\end{array}$} & \multicolumn{2}{|c|}{$\begin{array}{l}\text { 8-week WR } \\
\text { phase }\end{array}$} & \multicolumn{2}{|c|}{ Baseline } & \multicolumn{2}{|c|}{$\begin{array}{l}\text { 8-week WR } \\
\text { phase }\end{array}$} & \multicolumn{2}{|c|}{$\begin{array}{l}\text { 8-week BR } \\
\text { phase }\end{array}$} \\
\hline & Mean & SD & Mean & SD & Mean & SD & Mean & SD & Mean & SD & Mean & SD \\
\hline Body weight (kg) & $76 \cdot 4$ & $11 \cdot 2$ & $74 \cdot 7^{\star}$ & $10 \cdot 6$ & $76 \cdot 6$ & $10 \cdot 8$ & $76 \cdot 8$ & $15 \cdot 4$ & $77 \cdot 2$ & $15 \cdot 6$ & $76 \cdot 4$ & $16 \cdot 1$ \\
\hline BMI $\left(\mathrm{kg} / \mathrm{m}^{2}\right)$ & $26 \cdot 7$ & $2 \cdot 8$ & $26 \cdot 1^{*}$ & $2 \cdot 7$ & $26 \cdot 1$ & 2.7 & $26 \cdot 7$ & $4 \cdot 2$ & $26 \cdot 9$ & $4 \cdot 3$ & $26 \cdot 6$ & 4.5 \\
\hline Waist circumference $(\mathrm{cm})$ & $93 \cdot 2$ & $9 \cdot 2$ & $91 \cdot 7^{\star}$ & $8 \cdot 8$ & 91.5 & $8 \cdot 8$ & $92 \cdot 1$ & $9 \cdot 3$ & $92 \cdot 3$ & $11 \cdot 0$ & $90 \cdot 3^{*}$ & $10 \cdot 3$ \\
\hline Systolic blood pressure (mmHg) & 140 & 14 & $132^{*}$ & 11 & 139 & 21 & 141 & 13 & 140 & 16 & $134^{\star}$ & 13 \\
\hline Diastolic blood pressure (mmHg) & 84 & 11 & 86 & 9 & 88 & 12 & 85 & 8 & 89 & 9 & 88 & 8 \\
\hline Heart rate (beats/min) & 66 & 8 & 70 & 8 & 70 & 7 & 72 & 10 & 73 & 9 & 75 & 12 \\
\hline
\end{tabular}

$\mathrm{BR}$, brown rice; WR, white rice.

${ }^{\star}$ Mean value was significantly different from that at baseline $(P<0.05)$.

\section{Chronic effects}

Metabolic parameters. A few trials have examined the differential effects of whole and refined grains on body weight and weight changes. A 16-week clinical trial in Korean men with CHD has shown that the isoenergetic replacement of WR with whole grains and legume powder leads to significant reductions in serum glucose and insulin concentrations, whereas the body weight remains unchanged ${ }^{(29)}$. A study on Chinese type 2 diabetes mellitus patients has found that substituting BR with WR for 16 weeks did not substantially affect the concentrations of metabolic markers, although HDL-cholesterol levels and diastolic blood pressure were improved $^{(30)}$. In the present study, switching the staple food of the subjects to BR resulted in a significant decrease in body weight, BMI and waist circumference in those with the metabolic syndrome. There is a discrepancy between previous reports ${ }^{(29,30)}$ and the present one with regard to the effects of whole and refined grains on body weights, which may have been the result of differences in the study subjects. The beneficial effect of BR on body weight has been confirmed by a reduction in VFA, measured by abdominal computed tomography. Thus, changes in VFA $(\Delta V F A \%)$ were significantly lower after 8 weeks of BR consumption than after a comparable period of WR consumption among subjects in both the BRWR and WR-BR groups; however, changes in SFA $(\triangle \mathrm{SFA} \%)$ were comparable among the subjects, regardless of the staple food included in their diets. Previously, an analysis of fat distribution by computed tomography has demonstrated that visceral fat is decreased to a greater extent as a result of a low-energy diet than abdominal subcutaneous fat, particularly in subjects with visceral fat obesity ${ }^{(31)}$. These observations suggest that subjects with visceral fat obesity might be more susceptible to body weight reductions resulting from a diet including whole grains, including $\mathrm{BR}$.

There are three potential mechanisms by which BR decreases body weight and visceral fat in subjects with the metabolic syndrome. First, the lowered postprandial glucose and insulin levels associated with BR intake may lead to weight loss. Indirect evidence from both epidemiological and short-term experimental studies suggests the potential role of a high-GI diet, containing refined grains, in the development of obesity ${ }^{(32,33)}$. Alternatively, the low postprandial glucose and insulin levels associated with high whole grain intake may lead to weight loss, especially among overweight or obese individuals ${ }^{(34)}$. Reductions in postprandial glucose and insulin levels by an $\alpha$-glycosidase inhibitor, miglitol, have been shown to be associated with reductions in body weight, waist circumference and VFA in subjects with the metabolic syndrome ${ }^{(35)}$. Together, the lower postprandial glucose and insulin levels associated with BR intake may lead to weight loss in subjects with the metabolic syndrome. Second, alterations in hunger and/or increased satiety after BR consumption may lead to voluntary energy intake reductions. The consumption of low-GI foods has been reported to be directly associated with reductions in subsequent hunger and/or increased satiety, leading to low energy intake ${ }^{(36)}$. Although most of these trials were conducted over only a single meal or a single day, they collectively suggest that long-term consumption of whole-grain products may increase satiety and reduce energy consumption. Thus, a whole graincontaining diet may contribute to weight loss, especially in sedentary and overweight subjects. Epidemiological studies on dietary fibre consumption have also suggested that intake of whole grains is inversely associated with body weight and fat distribution ${ }^{(32,37)}$. The inherent high fibre content of whole-grain foods may help prevent weight gain by increasing appetite control through delayed carbohydrate absorption ${ }^{(38)}$. The correlation between dietary fibre and GI is modest, suggesting that other factors are important in determining the glycaemic effects of foods ${ }^{(38)}$. In the present study, the satiety scores recorded after consumption of meals with either BR or WR were comparable (Supplementary material 4, available online). The effects of BR on eating behaviour cannot be ruled out because the assessment of appetite and eating behaviour in humans is complicated and difficult ${ }^{(36)}$. The association between hunger and/or satiety and alteration of metabolic parameters should be evaluated in future studies. Third, microgradients, which are removed along with the outer bran by refining, may affect eating behaviour and insulin sensitisation independently by lowering the GI. Whole grains, including BR, are generally low in saturated fat and high in microgradients that might be associated with improved insulin sensitivity in metabolic derangement ${ }^{(4-6)}$. Since the 
Table 3. Changes in blood biochemical parameters (study 2)

(Mean values and standard deviations)

\begin{tabular}{|c|c|c|c|c|c|c|c|c|c|c|c|c|}
\hline \multirow[b]{3}{*}{ Parameters } & \multicolumn{6}{|c|}{ BR-WR group ( $n$ 14) } & \multicolumn{6}{|c|}{ WR-BR group ( $n$ 13) } \\
\hline & \multicolumn{2}{|c|}{ Baseline } & \multicolumn{2}{|c|}{ 8-week BR phase } & \multicolumn{2}{|c|}{ 8-week WR phase } & \multicolumn{2}{|c|}{ Baseline } & \multicolumn{2}{|c|}{ 8-week WR phase } & \multicolumn{2}{|c|}{ 8-week BR phase } \\
\hline & Mean & SD & Mean & SD & Mean & SD & Mean & SD & Mean & SD & Mean & SD \\
\hline \multicolumn{13}{|l|}{ Glucose (mmol/l) } \\
\hline 0 & $6 \cdot 2$ & 1.1 & 6 & 1 & 6 & 0.9 & 6 & 1.1 & 6.7 & $2 \cdot 6$ & $6 \cdot 2$ & 2.3 \\
\hline 30 & 9.5 & 2 & $9 \cdot 3$ & 1.8 & $9 \cdot 1$ & 1.9 & 9.0 & 1.9 & $10 \cdot 4$ & 3.5 & $10 \cdot 0$ & 3.4 \\
\hline 60 & 9.9 & $2 \cdot 9$ & $10 \cdot 6$ & $2 \cdot 9$ & 9.6 & 3.2 & $10 \cdot 6$ & $3 \cdot 7$ & 11.6 & 4.9 & $11 \cdot 1$ & $5 \cdot 6$ \\
\hline 120 & $8 \cdot 3$ & 3.5 & 8.5 & $2 \cdot 7$ & 8.4 & $2 \cdot 7$ & 9.0 & 3.6 & 9.9 & 5.4 & $9 \cdot 3$ & $5 \cdot 6$ \\
\hline \multicolumn{13}{|l|}{ Insulin (pmol//) } \\
\hline 0 & 62 & 35 & 59 & 25 & 62 & 26 & 58 & 22 & 82 & 53 & 77 & 47 \\
\hline 30 & 554 & 475 & 443 & 361 & 562 & 527 & 449 & 298 & 491 & 369 & 506 & 322 \\
\hline 60 & 547 & 437 & 547 & 295 & 495 & 282 & 583 & 375 & 665 & 460 & 567 & 238 \\
\hline 120 & 352 & 217 & 487 & 352 & 520 & 324 & 463 & 225 & 476 & 271 & 360 & 152 \\
\hline HOMA-IR & 2.89 & 1.40 & $2 \cdot 23^{\star}$ & 0.95 & 2.51 & 1.33 & 2.79 & 1.24 & 3.83 & $8 \cdot 10$ & $2.34 \dagger$ & 1.75 \\
\hline HOMA- $\beta$ & 75 & 43 & 77 & 50 & 77 & 46 & 83 & 41 & 96 & 63 & 106 & 83 \\
\hline $\mathrm{HbA}_{1 \mathrm{c}}(\mathrm{NGSP} \%)$ & 5.74 & 0.55 & 5.72 & 0.45 & 5.9 & 1.55 & $5 \cdot 78$ & 0.81 & 5.83 & 1.43 & $6 \cdot 19$ & 1.55 \\
\hline Total cholesterol $(\mathrm{mmol} / \mathrm{l})$ & 5.85 & 0.54 & $5 \cdot 31^{*}$ & 0.40 & $5 \cdot 66$ & 0.72 & 5.67 & 0.68 & $5 \cdot 27$ & 0.61 & 5.56 & 0.54 \\
\hline LDL-cholesterol (mmol/l) & 3.45 & 0.40 & $3 \cdot 15^{\star}$ & 0.35 & 3.37 & 0.56 & 3.48 & 0.52 & $3 \cdot 28$ & 0.48 & 3.41 & 0.59 \\
\hline HDL-cholesterol (mmol/l) & 1.44 & 0.30 & 1.37 & 0.23 & 1.47 & 0.24 & 1.26 & 0.24 & $1 \cdot 21$ & 0.19 & $1 \cdot 20$ & 0.14 \\
\hline $\mathrm{TAG}(\mathrm{mmol} / \mathrm{l})$ & 2.05 & 1.07 & 1.69 & 0.65 & $1 \cdot 74$ & 0.65 & 1.95 & 0.90 & 1.65 & 0.58 & 1.42 & 0.50 \\
\hline NEFA $(\mathrm{mmol} / \mathrm{l})$ & 0.13 & 0.08 & 0.11 & 0.05 & 0.15 & 0.14 & 0.09 & 0.04 & 0.13 & 0.19 & 0.12 & 0.12 \\
\hline AST (IU/I) & 25 & 8 & 27 & 21 & 24 & 8 & 23 & 6 & 24 & 9 & 25 & 9 \\
\hline ALT (IU/I) & 32 & 19 & 40 & 38 & 31 & 16 & 36 & 16 & 42 & 21 & 43 & 21 \\
\hline$\gamma$-GTP (IU/I) & 53 & 24 & 64 & 31 & 54 & 29 & 67 & 36 & 68 & 35 & 70 & 45 \\
\hline Uric acid (mg/l) & $66 \cdot 3$ & $9 \cdot 7$ & $70 \cdot 1$ & $10 \cdot 8$ & $68 \cdot 2$ & 9.5 & 68 & $15 \cdot 7$ & $65 \cdot 8$ & $16 \cdot 4$ & 64.6 & $13 \cdot 0$ \\
\hline High-sensitivity CRP (mg/l) & 0.9 & 0.4 & $1 \cdot 1$ & 0.9 & 0.8 & 0.5 & $1 \cdot 1$ & 0.4 & $2 \cdot 3$ & 1.6 & 1.3 & 0.9 \\
\hline GLP-1 (pmol/l) & $2 \cdot 17$ & 0.41 & $2 \cdot 41$ & 0.79 & $2 \cdot 29$ & 0.49 & $2 \cdot 80$ & 0.14 & 3.26 & 1.55 & 3.81 & $2 \cdot 12$ \\
\hline Urinary 8-isoprostane (ng/ml) & 112 & 25 & 132 & 90 & 109 & 47 & 148 & 84 & 164 & 93 & 112 & 42 \\
\hline Urinary albumin excretion (mg/g creatinine) & 4.04 & 2.39 & 4.59 & 4.66 & $5 \cdot 16$ & 8.24 & 3.73 & 1.72 & $5 \cdot 42$ & 4.05 & $6 \cdot 30$ & $8 \cdot 13$ \\
\hline
\end{tabular}

BR, brown rice; WR, white rice; HOMA-IR, homeostasis model assessment of insulin resistance; $\mathrm{HbA}_{1 \mathrm{c}}$, glycosylated $\mathrm{Hb}$; NGSP, National Glycohemoglobin Standardization Program; AST, aspartate aminotransferase; ALT, alanine transaminase; $\gamma$-GTP, $\gamma$-glutamyl transpeptidase; CRP, C-reactive protein; GLP-1, glucagon-like peptide-1.

"Mean value was significantly different from that at baseline $(P<0.05)$

† Mean value was significantly different from that of the 8-week WR phase. 

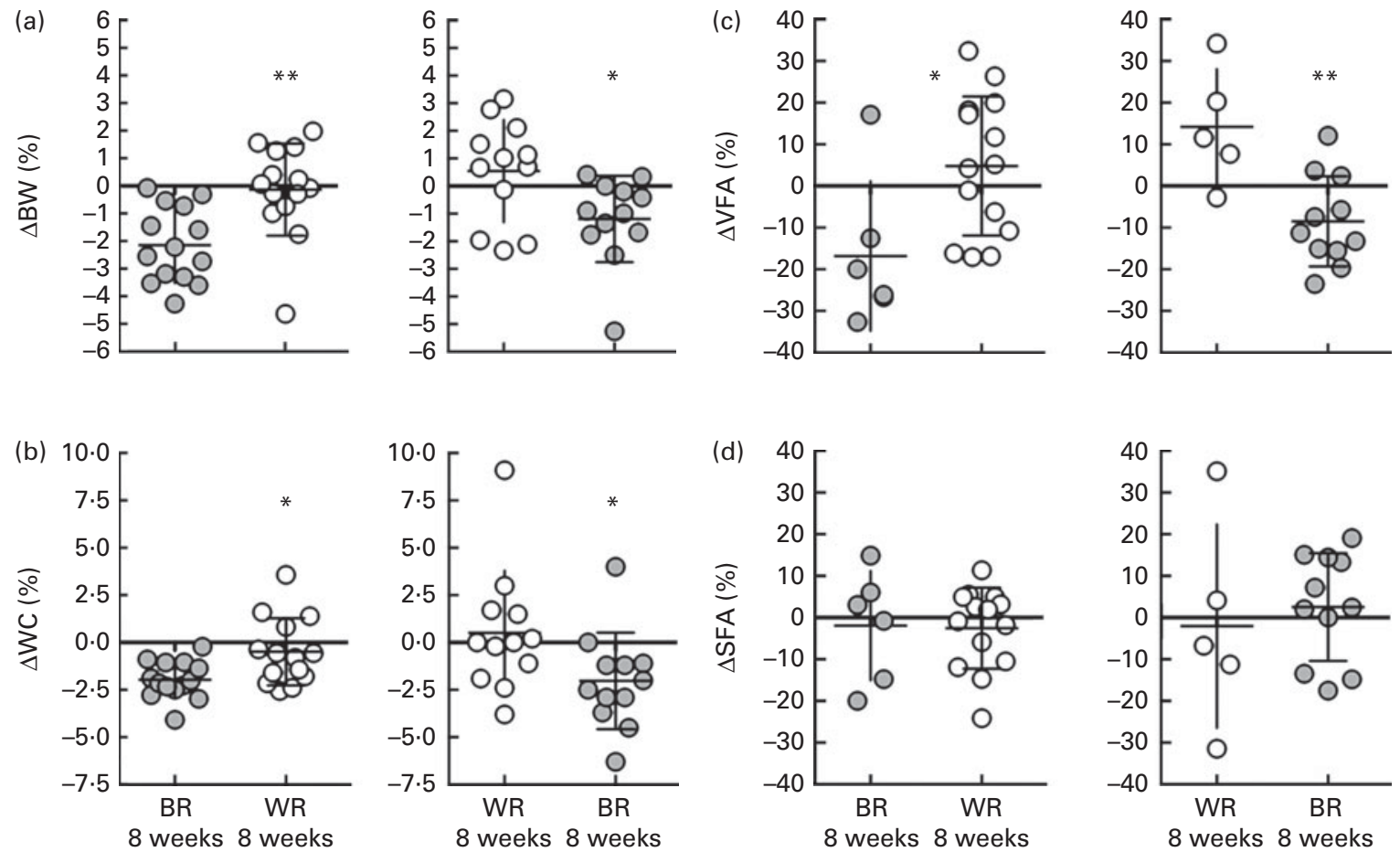

Fig. 3. Percentage changes in (a) body weight $(\triangle B W)$, (b) waist circumference $(\triangle W C)$, (c) visceral fat area $(\triangle V F A)$ and (d) subcutaneous fat area $(\triangle S F A)$ after ingestion of a brown rice (BR) or a white rice (WR) diet in obese subjects. Obese participants (BMI $\geq 25 \mathrm{~kg} / \mathrm{m}^{2}$ ) were randomised to either a diet including $B R$ followed by a diet including WR group (BR-WR, $n$ 14) or a WR-containing diet followed by the one containing BR group (WR-BR, $n$ 13). Before and after completion of the first and second 8-week terms, blood and urine samples were collected, and abdominal fat computed tomography scans were taken. Values represent percentage change from baseline values of each 8-week term. Values are means, with standard deviations represented by vertical bars. (a) Mean value was significantly different from that at baseline following the consumption of the BR diet: $P=0.009, P=0.045$ (paired $t$ test). (b) Mean value was significantly different from that at baseline following the consumption of the BR diet: $P=0.032, P=0.047$ (paired $t$ test). (c) Mean value was significantly different from that following the consumption of the BR diet: $P=0.018, P=0.003$ (unpaired $t$ test). Mean value was significantly different from that at baseline: ${ }^{*} P<0.05,{ }^{* *} P<0.01$.

concentrations of markers of radical oxygen species (isoprostane), inflammation (hs-CRP) and incretin (active GLP-1) were all comparable between the BR and WR diet groups in the present study, other mechanisms must be involved in the observed improvements in obesity and insulin sensitivity. Recently, we have reported that BR and its component, $\gamma$-oryzanol, alter eating behaviour and fuel homeostasis in mice ${ }^{(7)}$. When mice were allowed free access to both a BRcontaining chow diet and a high-fat diet, they preferred the chow diet to the high-fat diet. BR and $\gamma$-oryzanol improve high-fat diet-induced metabolic derangement and attenuate the preference for dietary fat by decreasing hypothalamic endoplasmic reticulum stress. The relevance of microgradients, such as $\gamma$-oryzanol, to eating behaviour needs to be validated in future human studies.

Endothelial function. As shown in Fig. 4, FMD values (\%) were increased from baseline after consumption of the BR diet for 8 weeks, but returned to baseline values after consumption of WR for a similar period of time. NMD values (\%) were comparable with those at baseline after consumption of diets containing either type of rice. Decreased FMD has been reported to be associated with cardiovascular risk factors, including obesity and the metabolic syndrome, and with the estimated 10-year risk of $\mathrm{CHD}^{(20,21)}$. Interventions to reduce cardiovascular risk in adults have demonstrated a parallel improvement in $\operatorname{FMD}^{(20,21)}$. These observations led to the postulation of three mechanisms by which BR may improve FMD. First, the effects of BR on postprandial glucose and insulin levels may be associated with improved endothelial function ${ }^{(39)}$, as suggested by the association of postprandial
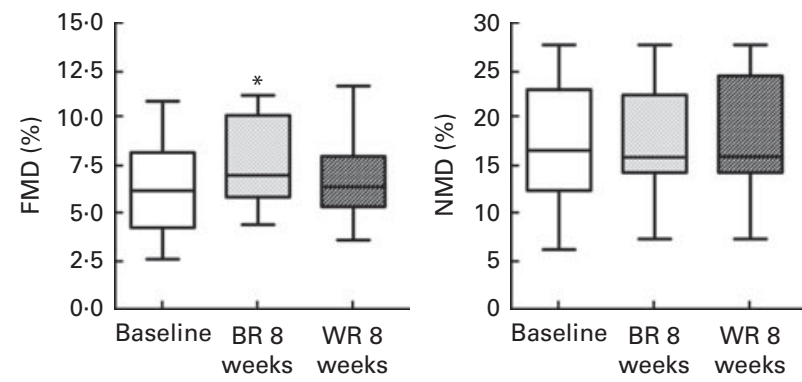

Fig. 4. Changes in forearm flow-mediated dilation (FMD) and nitroglycerinemediated dilation (NMD) values after brown rice (BR) or white rice (WR) diet consumption in obese subjects. Obese participants (BMl $\geq 25 \mathrm{~kg} / \mathrm{m}^{2}$ ) were randomised into either a group consuming a diet that included BR for an 8-week period, followed by consumption of a diet containing WR for a similar period (BR-WR, $n$ 14), or a group consuming the diet in the reverse order (WR-BR, $n$ 13). Before and after completion of each 8-week term, FMD and NMD were measured using a novel vascular ultrasound system equipped with an edge-tracking system for two-dimensional imaging and automatic measurement. FMD and NMD values were calculated as follows: FMD or NMD value $(\%)=($ maximum diameter - diameter at rest $) \times 100 /$ diameter at rest. Values are means with minimum and maximum values, with standard deviations represented by vertical bars. ${ }^{*}$ Mean value was significantly different from that at baseline $(P<0.05$; one-way ANOVA or paired $t$ test). 
glucose levels with macrovascular complications ${ }^{(11,14)}$. Postprandial glucose elevation has also been suggested to elicit endothelial dysfunction ${ }^{(12,13)}$, a surrogate marker of future cardiovascular events ${ }^{(12-15)}$. Second, a reduction in body weight and/or improved insulin sensitivity may be associated with improved endothelial function. After 8 weeks of BR consumption, there was a significant negative correlation with HOMA-IR and a borderline correlation with VFA. However, the effects of body weight reduction on endothelial function have been inconclusive. FMD was not improved with drug-induced weight loss in overweight adults ${ }^{(40)}$, whereas another study has shown increased FMD with the use of a lipase inhibitor (orlistat) that prevents fat absorption $^{(41)}$. In a double-blind, placebo-controlled study investigating the effect of orlistat, Bergholm et al. ${ }^{(42)}$ demonstrated that the lowering of LDL-cholesterol levels, rather than moderate weight loss, improved endothelial function in obese subjects. The weight loss associated with a very low-energy, 2-week diet has been reported to improve endothelium-dependent vasodilation in obese, hypertensive subjects ${ }^{(43)}$. Moreover, dietary changes, combined with exercise, have been found to elicit a multiplicative response, and improvements in FMD appeared to be independent of improvements in glucose tolerance ${ }^{(44)}$. The attenuation of insulin resistance, rather than changes in carbohydrate tolerance, might be more important in affecting improvement in endothelial function ${ }^{(45)}$. Third, a reduction in LDL-cholesterol levels and blood pressure may directly affect endothelial function. The concentrations of markers of radical oxygen species (isoprostane), inflammation (hs-CRP) and incretin (active GLP-1) were unchanged, but LDL-cholesterol levels and systolic blood pressure were improved by BR ingestion.

As has been discussed above, the underlying mechanism(s) by which BR improves endothelial function may be multiactorial. Further studies are required to confirm our observations and clarify the mechanism(s) underlying endothelial function improvement. An inverse relationship between the intake of whole grains and the risk of IHD has been reported by large cohort studies ${ }^{(9,10)}$. If the above-mentioned beneficial effects of BR on endothelium are elicited in a clinical setting, then BR may provide protection against atherosclerotic cardiovascular events, in part, by improving endothelial function.

\section{Limitations}

The present study has several limitations. First, the number of patients was too small for any definite conclusions to be drawn, especially in study 1 . Second, the study could not determine a causal relationship between BR consumption and endothelial function or weight loss. Third, the present study includes potential confounding factors that may have affected the observed effects of daily BR or WR consumption. This was because it was not possible to closely and individually match side foods, based on macronutrient composition and palatability, among the study subjects. Lastly, the assessments of hunger and satiety were not precise. Although the duration of satiety might be prolonged by $\mathrm{BR}$, changes in eating behaviour, manifest by hunger and satiety, could not be quantified or characterised.

\section{Conclusion}

The results of the present study suggest that BR could be beneficial, partly through lowering of the postprandial glycaemic response, and may provide a measure of protection to postprandial endothelial function in subjects with the metabolic syndrome. Long-term benefits of BR on metabolic parameters and endothelial function were also observed. Future studies with larger cohort sizes and longer durations of follow-up are warranted to examine the effects of substituting BR with WR on metabolic risk for future cardiovascular events.

\section{Supplementary material}

To view supplementary material for this article, please visit http://dx.doi.org/0.1017/S0007114513002432

\section{Acknowledgements}

The authors cordially acknowledge the staff of Tomishiro Central Hospital, Okinawa, Japan, especially Kaori Ichimatsu, at the Nutrition Division, for the diet protocol and Hiroe Shinjo, Saeko Nakamura, Ayano Kamiyama, Merina Ohta and Megumi Yonaha, at the Diabetes and Lifestyle-related Disease Center, for their devoted secretarial work and Hiroyuki Oshiro, Okinawa Shokuryo K.K., Okinawa, Japan for BR and WR. The present study was supported by grants from the Ministry of Education, Culture, Sports, Science and Technology (MEXT) and the Ministry of Health, Labour and Welfare (MHLW), Japan. MEXT and MHLW had no role in the design, analysis or writing of this article. The authors' contributions are as follows: Mi. S. designed the present study, analysed the data and wrote the manuscript; $M$. $H$. was involved in patient management, data collection and discussion; R. K. performed the vascular function study; Ke. Y., H. T., C. K., Ko. Y., S. T., Ma. S. and H. M. contributed to the discussion.

None of the authors has any conflicts of interest.

\section{References}

1. O'Dea K, Nestel PJ \& Antonoff L (1980) Physical factors influencing postprandial glucose and insulin responses to starch. Am J Clin Nutr 33, 760-765.

2. Jenkins DJ, Wolever TM, Taylor RH, et al. (1981) Glycaemic index of foods: a physiological basis for carbohydrate exchange. Am J Clin Nutr 34, 362-366.

3. Foster-Powell K, Holt SH \& Brand-Miller JC (2002) International table of glycaemic index and glycaemic load values. Am J Clin Nutr 76, 5-56.

4. McKeown NM, Meigs JB, Liu S, et al. (2004) Carbohydrate nutrition, insulin resistance, and the prevalence of the metabolic syndrome in the Framingham Offspring Cohort. Diabetes Care 27, 538-546.

5. Tighe P, Duthie G, Vaughan N, et al. (2010) Effect of increased consumption of whole-grain foods on blood pressure and other cardiovascular risk markers in healthy 
middle-aged persons: a randomized controlled trial. Am J Clin Nutr 92, 733-740.

6. Nettleton JA, McKeown NM, Kanoni S, et al. (2010) Interactions of dietary whole-grain intake with fasting glucoseand insulin-related genetic loci in individuals of European descent: a meta-analysis of 14 cohort studies. Diabetes Care 33, 2684-2691.

7. Kozuka C, Yabiku K, Sunagawa S, et al. (2012) Brown rice and its component, $\boldsymbol{\gamma}$-oryzanol, attenuate the preference for high-fat diet by decreasing hypothalamic endoplasmic reticulum stress in mice. Diabetes 61, 3084-3093.

8. Jacobs DR Jr, Meyer KA, Kushi LH, et al. (1998) Whole-grain intake may reduce the risk of ischemic heart disease death in postmenopausal women: the Iowa Women's Health Study. Am J Clin Nutr 68, 248-257.

9. Liu S, Stampfer M, Hu F, et al. (1999) Whole grain consumption and risk of coronary heart disease: results from the Nurses' Health Study. Am J Clin Nutr 70, 412-419.

10. Ma XY, Liu JP \& Song ZY (2012) Glycemic load, glycemic index and risk of cardiovascular diseases: meta-analyses of prospective studies. Atherosclerosis 223, 491-496.

11. Coutinho M, Gerstein HC, Wang Y, et al. (1999) The relationship between glucose and incident cardiovascular events. A metaregression analysis of published data from 20 studies of 95,783 individuals followed for 12.4 years. Diabetes Care 22, $233-240$.

12. Kawano H, Motoyama T, Hirashima O, et al. (1999) Hyperglycemia rapidly suppresses flow-mediated endotheliumdependent vasodilation of brachial artery. J Am Coll Cardiol 34, 146-154.

13. Shimabukuro M, Chinen I, Higa N, et al. (2007) Effects of dietary composition on postprandial endothelial function and adiponectin concentrations in healthy humans: a crossover controlled study. Am J Clin Nutr 86, 923-928.

14. Ceriello A, Hanefeld M, Leiter L, et al. (2004) Postprandial glucose regulation and diabetic complications. Arch Intern Med 164, 2090-2095.

15. Jonk AM, Houben AJ, Schaper NC, et al. (2011) Obesity is associated with impaired endothelial function in the postprandial state. Microvasc Res 82, 423-429.

16. Seino Y, Nanjo K, Tajima N, et al. (2010) Report of the Committee on the classification and diagnostic criteria of diabetes mellitus. J Diab Invest 1, 212-228.

17. Alberti KG, Zimmet P \& Shaw J (2006) Metabolic syndrome - a new world-wide definition. A Consensus Statement from the International Diabetes Federation. Diabet Med 23, 469-480.

18. Fujita K, Nishizawa H, Funahashi T, et al. (2006) Systemic oxidative stress is associated with visceral fat accumulation and the metabolic syndrome. Circ J 70, 1437-1442.

19. Faul F, Erdfelder E, Buchner A, et al. (2009) Statistical power analyses using G*Power 3.1: tests for correlation and regression analyses. Behav Res Methods 41, 1149-1160.

20. Corretti MC, Anderson TJ, Benjamin EJ, et al. (2002) Guidelines for the ultrasound assessment of endothelial-dependent flow-mediated vasodilation of the brachial artery: a report of the International Brachial Artery Reactivity Task Force. J Am Coll Cardiol 39, 257-265.

21. Inoue T, Matsuoka H, Higashi Y, et al. (2008) Flow-mediated vasodilation as a diagnostic modality for vascular failure. Hypertens Res 31, 2105-2113.

22. Friedewald WT, Levy RI \& Fredrickson DS (1972) Estimation of the concentration of low-density lipoprotein cholesterol in plasma, without use of the preparative ultracentrifuge. Clin Chem 18, 499-502.

23. The Committee of Japan Diabetes Society on the Diagnostic Criteria of Diabetes Mellitus (2010) Report of the Committee on the classification and diagnostic criteria diabetes mellitus. J Japan Diab Soc 53, 450-467.

24. Mannucci E, Ognibene A, Cremasco F, et al. (2001) Effect of metformin on glucagon-like peptide 1 (GLP-1) and leptin levels in obese nondiabetic subjects. Diabetes Care $\mathbf{2 4}$ 489-494.

25. Jenkins DJ, Wesson V, Wolever TM, et al. (1988) Wholemeal versus wholegrain breads: proportion of whole or cracked grain and the glycaemic response. BMJ 297, 958-960.

26. Ito Y, Mizukuchi A, Kise M, et al. (2005) Postprandial blood glucose and insulin responses to pre-germinated brown rice in healthy subjects. J Med Invest 52, 159-164.

27. Pereira MA, Jacobs DR Jr, Pins JJ, et al. (2002) Effect of whole grains on insulin sensitivity in overweight hyperinsulinemic adults. Am J Clin Nutr 75, 848-855.

28. Shimabukuro M, Higa N, Chinen I, et al. (2006) Effects of a single administration of acarbose on postprandial glucose excursion and endothelial dysfunction in type 2 diabetic patients: a randomized cross-over study. J Clin Endocrinol Metab 91, 837-842.

29. Jang Y, Lee JH, Kim OY, et al. (2001) Consumption of whole grain and legume powder reduces insulin demand, lipid peroxidation, and plasma homocysteine concentrations in patients with coronary artery disease: randomized controlled clinical trial. Arterioscler Thromb Vasc Biol 21, 2065-2071.

30. Zhang G, Pan A, Zong G, et al. (2011) Substituting white rice with brown rice for 16 weeks did not substantially affect metabolic risk factors in middle-aged Chinese men and women with diabetes or a high risk for diabetes. $J$ Nutr 141, 1685-1690.

31. Fujioka S, Matsuzawa Y, Tokunaga K, et al. (1991) Improvement of glucose and lipid metabolism associated with selective reduction of intra-abdominal visceral fat in premenopausal women with visceral fat obesity. Int $J$ Obes $\mathbf{1 5}$, 853-859.

32. Ludwig DS, Pereira MA, Kroenke CH, et al. (1999) Dietary fiber, weight gain, and cardio-vascular disease risk factors in young adults. J Am Med Assoc 282, 1539-1546.

33. Thomas DE, Elliott EJ \& Baur L (2007) Low glycaemic index or low glycaemic load diets for overweight and obesity. The Cochrane Database of Systematic Reviews 2007 issue 3, CD005105.

34. McKeown NM, Meigs JB, Liu S, et al. (2004) Carbohydrate nutrition, insulin resistance, and the prevalence of the metabolic syndrome in the Framingham Offspring Cohort. Diabetes Care 27, 538-546.

35. Shimabukuro M, Higa M, Yamakawa K, et al. (2012) Miglitol, $\alpha$-glycosidase inhibitor, reduces visceral fat accumulation and cardiovascular risk factors in subjects with the metabolic syndrome: a randomized comparable study. Int J Cardiol (epublication ahead of print version 19 June 2012).

36. Niwano Y, Adachi T, Kashimura J, et al. (2009) Is glycaemic index of food a feasible predictor of appetite, hunger, and satiety? J Nutr Sci Vitaminol 55, 201-207.

37. Fukagawa NK, Anderson JW, Hageman G, et al. (1990) Highcarbohydrate, high-fiber diets increase peripheral insulin sensitivity in healthy young and old adults. Am J Clin Nutr 52, 524-528.

38. Koh-Banerjee P \& Rimm EB (2003) Whole grain consumption and weight gain: a review of the epidemiological evidence, potential mechanisms and opportunities for future research. Proc Nutr Soc 62, 25-29.

39. Adolphe JL, Drew MD, Huang Q, et al. (2012) Postprandial impairment of flow-mediated dilation and elevated methylglyoxal after simple but not complex carbohydrate consumption in dogs. Nutr Res 32, 278-284. 
40. Brook RD, Bard RL, Glazewski L, et al. (2004) Effect of short-term weight loss on the metabolic syndrome and conduit vascular endothelial function in overweight adults. Am J Cardiol 93, 1012-1016.

41. Sekuri C, Tavli T, Avsar A, et al. (2003) The acute effect of orlistat on endothelial function in young obese women. Int J Clin Pharmacol Res 23, 111-117.

42. Bergholm R, Tiikkainen M, Vehkavaara S, et al. (2003) Lowering of LDL cholesterol rather than moderate weight loss improves endothelium-dependent vasodilation in obese women with previous gestational diabetes. Diabetes Care 26, 1667-1672.
43. Sasaki S, Higashi Y, Nakagawa K, et al. (2002) A low-calorie diet improves endothelium-dependent vasodilation in obese patients with essential hypertension. Am J Hypertens 15, 302-309.

44. Hamdy O, Ledbury S, Mullooly C, et al. (2003) Lifestyle modification improves endothelial function in obese subjects with the insulin resistance syndrome. Diabetes Care 26, $2119-2125$.

45. Shimabukuro M (2009) Cardiac adiposity and global cardiometabolic risk: new concept and clinical implication. Circ $J$ 73, 27-34. 